\section{Military Technical College Kobry El-Kobbah, Cairo, Egypt.}

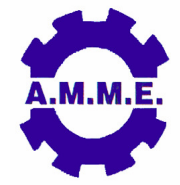

\title{
SWIRL GRANULAR FLOWS IN A HORIZONTAL SHAKEN GRANULAR MATERIAL
}

A. Bakhshinejad* and P. Zamankhan**

\begin{abstract}
A series of experiments done in which a granular material is exposed to horizontal vibration within a rectangular container with two hallow cylinders mounted inside of it. At high values of the peak acceleration relative to gravity novel swirl granular flows are observed within the cylinders while the grains cascade down the outer surface of the piles which fixed in the bottom of the container within the rectangular container outside the cylinders. Computer simulations are performed which may support the existent interpretation of the unusual aforementioned behaviour.
\end{abstract}

\section{KEY WORDS}

Swirl granular flows, horizontal vibration, instabilities and computer simulations.

* Graduate student, Faculty of Industrial, Mechanical Engineering and Computer Sciences, University of Iceland.

** Professor, Faculty of Industrial, Mechanical Engineering and Computer Sciences, University of Iceland. 


\section{INTRODUCTION}

Granular materials are abundant in nature and their vibration plays an important role in natural events such as earthquakes [1] and avalanches [2]. Vibration of granular materials is commonly used in industrial devices as an aid to handle grains in many practical applications such as drying, powder mixing, and separation processes [3]. In industrial situations, horizontal vibrations are used in a number of applications including industrial vibrating screens. There are few studies which focus on horizontal vibrations [4]. When a bed of fluid-immersed grains is exposed to horizontal vibration, which is held upon a horizontal container, solid-fluid granular transitions can be observed [5]. Metcalfe et al. [5] studied solid-fluid granular transitions in a horizontally vibrated container of granular materials and observed hysteresis during the transition. They also observed that a modest air flow corresponds to about $40 \%$ of the weight of the bed which effectively removes the hysteresis in the initial transition. Rubin et al. [6] measured the steady slope of the shaped granular material under the influence of horizontal vibration using optical particle tracking. The optical particle tacking is a technique to determine the motion of particles using the reflected light from the surface particles. A fast speed camera records the optical motion and image processing software analyzes the vectorial orientation of the particles. [7] The motion of the beads was found to be intermittent which indicates that strengthening micro-rearrangements are important features that affect failure of slopes under external perturbations.

A horizontal granular bed becomes unstable and acquiring a tilt at the high peak acceleration relative to gravity, $\Gamma$, [8]. Aumaitre et al. [8] studied the onset and dynamics of flow in shallow horizontally oscillating granular layers as a function of the depth of the layer and imposed acceleration. They found out that for avalanches of inclined layers the thresholds for starting and stopping of flow are slightly different. At high the peak acceleration relative to gravity $\Gamma$ the motion was found to be fluidlike over the entire cycle. The measured flow profiles which had time-dependent shapes were found to be significantly different from the previously measured profiles for avalanche flows.

The dynamics and stability of a bed, composed of solid particles and immersed in a fluid, may be analysed using a technique developed by Anderson and Jackson [9] which might be more advantageous for modeling continuous flows [10]. Hence, an alternative approach in which Lagrangian (particle-based) and Eulerian (grid-based) methods $(\mathrm{Eu} / \mathrm{La})$ are combined is required to predict the transition between intermittent and continuous flow. In this approach, the gas dynamics are included using large-eddy simulations (LES) [11] while the dynamics of grains are described through molecular dynamics where the interaction between the surfaces of the grains is modelled using the generalized form of contact theory developed by Hertz [12]. In addition, the coefficient of kinetic friction is assumed to depend on the relative velocity of slipping [13].

This article describes the formation of tilts in shaken granular materials within a rectangular container which is being vibrated horizontally, as:

$$
r=A_{x} \sin \left(\omega_{x} t\right) e_{x}+A_{y} \cos \left(\omega_{y} t\right) e_{y}
$$


Here, $A_{x}, \omega_{x}, \mathbf{e}_{x}$ and $A_{y}, \omega_{y}, \mathbf{e}_{y}$ are the physical amplitudes of the vibrating container, the angular frequencies of the vibration, and the unit vectors in the $x$ - and $y$-direction, respectively. The container includes two hollow cylindrical vessels which are mounted on the inside surface of the container. Novel swirl granular flows are observed in the cylinders. In addition, the grains are seen to cascade down the outer surface of the piles within the rectangular container outside the cylinders.

This paper studies the swirl granular flows using numerical techniques. Note that conducting thorough investigations of the complex granular flows is not as simple without numerical simulations. The organization of this paper is as follows. In Sec. 2 the experimental setup is described and the findings of the experimental results are presented. Sec. 3 includes the mathematical model and results of numerical simulations for swirl granular flows. The results obtained by using GPU (graphics processing unit) technology fully satisfy the general purpose of scientific and engineering computing [14]. Finally, the conclusions are summarized in sec.4.

\section{EXPERIMENTS AND OBSERVATIONS}

\section{Experimental Setup}

Images of the polycarbonate plastic container used in this study, as well as some nomenclatures, are illustrated in Figs. 1 (a) and (b). The container, filled with sand particles, is fixed at the centre of a thin cardboard which in turn is clipped onto the bottom of the rectangular pad of an orbital shaker. The physical and mechanical properties of polycarbonate plastics are given in Table 1.

Orbital shakers have widely been used in pharmaceutical industries to mix the liquids in biopharmaceutical processes [15]. In addition, orbital shakers are used to study the aggregation and breakup of microorganisms and inorganic particle flocks in water [16].

A function generator is used to provide a sinusoidal signal of adjustable frequency and amplitude to a power amplifier which in turn amplifies and transmits the input signal to the orbital shaker. The accelerations of vibration in the $x$ - and $y$-direction are monitored by a 3-axis accelerometer attached to the pad to correctly measure the vibrations. The accelerometer is based on MEMS sensors. The sensors have a sensitivity of $100 \mathrm{mV} / \mathrm{g}$ with high resolution measurement up to $\pm 16 \mathrm{~g}$. A data acquisition system acquired the measured acceleration from the accelerometer and transferred the digitized output via a USB link to a computer for analysis.

The sand used in the experiments had a rough surface with a medium sphericity and high elasticity; the physical and mechanical properties are given in Table 1. A sieve analysis [17] was performed to assess the particle size distribution of sand. The results of the test are shown in graphical form in Fig. 1 (c) to identify the particle-size distribution (PSD) of sand. The circles in Fig.1 (c) represent the relative amounts of sorted particles according to size. The solid line in Fig. 1 (c) represents a model of the size distribution of sand. The model implies that the size distribution of the sand used in experiments is a logarithmic normal distribution given as 


$$
f_{x}\left(d_{p}^{*}\right)=\frac{1}{d_{p}^{*} \sigma \sqrt{2 \pi}} e^{-\frac{\left(\ln d_{p}^{*}-\mu\right)^{2}}{2 \sigma^{2}}}
$$

Where $d_{p}^{*}$ is the normalised particle size defined as, $d_{p}^{*}=d_{p} / d_{0}, d_{0}$ is the reference particle size, $\mu$ is the mean and $\sigma$ is the standard deviation. In this case, $d_{0}=400 \mu \mathrm{m}$, $\mu=0.69$ and $\sigma=0.68$.

Fig. 1 (d) shows a snapshot of a spill of the sand particles which is used in flat surface experiments. As can be seen from Fig. 1 (d), the grains are accumulated in a heap with a linear region characterized by the angle of repose $\theta_{z}$. The overall coefficient of static friction of the grains can be estimated as $\mu_{s}=\tan \left(\theta_{s}\right)=0.65$.

The shaker pad is subjected to horizontal oscillations of the form of equation (1). Fig. 1 (e) depicts the ellipse-type orbits which is a result of an offset weight being placed onto the motor shaft of the shaker at $\omega_{x} / 2 \pi=42 \mathrm{~Hz}$ and $A_{x}=10^{-3} \mathrm{~m}$ and $\omega_{y} / 2 \pi=25 \mathrm{~Hz}$ and $A_{y}=10^{-3} \mathrm{~m}$ which are corresponding to the values of $\Gamma_{x}=7.1$ and $\Gamma_{y}=2.5$, respectively. The shaker can operate at different angular speeds.

\section{Observations}

The aim is to determine the possibility of tilts in a rectangular container of the first set of experiments. In the case of fluidized bed coating, air is passed through a bed of sand particles. Initially the sand particles form a flat surface before they are exposed to horizontal vibrations at $\omega_{x} / 2 \pi=42 \mathrm{~Hz}$ and $A_{x}=10^{-3} \mathrm{~m}$ and $\omega_{y} / 2 \pi=25 \mathrm{~Hz}$, and $A_{y}=10^{-3} \mathrm{~m}$. When the frictional force, acting on the particles, or the pressure drop of the flowing air through the bed equals or exceeds the weight of the bed, the powder particles become suspended and the bed exhibits liquid-like behaviour. Figures 1 (a) and (b) illustrate instantaneous configurations after $t=5 \mathrm{~s}$. As can be seen from Figs. 1 (a) and (b), the bed geometry becomes unstable and the bed breaks up in a spatial symmetry by producing piles. The sand particles move down by horizontal vibration to the outer surface of the piles which are formed close to the container walls. The particles are rearranging in a trough which is directed from north to south in the middle of the container. Here, the north-south direction describes the $x$-axis.

It is well known that granular flows can be significantly influenced by the container geometry. To investigate the influence of the geometry, the second set of experiments is carried out in a foam container. In this experiment, we were interested to investigate air-particle interactions. So we did not use very large particles. We used sand with high coefficient of friction. The aim was to study role played by the friction. Figure 2 shows the images of a black foam tray with two hallow cylinders made out of glass inside of the tray. The container is filled with sand particles and fixed at the centre of a thin cardboard. The cardboard is clipped onto the bottom of a rectangular pad of the shaker. Beginning with an initially flat free surface, the sand particles are exposed to horizontal oscillations of the form of equation (1) where $\omega_{x} / 2 \pi=42 \mathrm{~Hz}$ and $A_{x}=10^{-3} \mathrm{~m}$ and $\omega_{y} / 2 \pi=25 \mathrm{~Hz}$, and 
$A_{y}=10^{-3} \mathrm{~m}$. The physical and mechanical properties of the foam and glass containers are given in Table. 1.

Swirl granular flows are formed within the cylinders while the grains cascade down the outer surface of the piles within the rectangular container outside of the cylinders. To quantify the spin of the sand particles on the top of the cylinders, a simple propeller shaft system is used as depicted in Fig. 2 (b-d). Figure 2 (d) shows a simple bearing. A shaft bearing assembly, as illustrated in Fig. 2 (c), is designed to allow the shaft to spin in the vertical direction relative to the cylindrical container. The plastic propeller is attached to the shaft as shown in Fig. 2 (c). The physical and mechanical properties of the plastic propeller shown in Fig. 2 (c) are given in Table. 1. Fig. 2 (e) illustrates an image of the spining propeller taken with a slow shutter speed. The speed of the propeller depends on a number of factors including the particle size and the shape of particles. Qualitative analyses of the image show a high spin of the sand particles. An accurate inspection of this image reveals that the spin of the sand particles is quite high. Images of the propeller and the container are captured and saved by using a frame grabber board connected to a video camera.

Mathematically, it is not possible to reconstruct a 3D model from a single image of the container. However, an optical processing algorithm can be developed that employs numerous photographs taken at multiple known fixed locations at approximately the same time to create a 3 dimensional model of the system as shown in Fig. 2. All position measurements are relative to a frame of reference at $\mathrm{t}=0$. By using target identification techniques and movement, the position would be back calculated to decrease the errors of the 3D model. An analysis of the images using the aforementioned image analysis technique, made it possible to estimate the average spin of the propeller in the $z$-direction which is $\omega=18 \mathrm{rad} / \mathrm{s}$. Apparently,

there is no study which focuses on swirl granular. In order to initial study of this new granular flow regime further, computer simulations of a granular material within a rectangular container, which is exposed to horizontal oscillations of the form of equation (1), are summarized in the next section.

\section{COMPUTER SIMULATIONS}

Figure 1 (c) reveals that the particle size of $d_{p} \approx 500 \mu m$ has the highest probability distribution. The size distribution and the shape of particles are important factors in granular flows which are described in the preceding section. The simulations are restricted to a mixture of spherical grains with diameters $d_{p 1}=500 \mu \mathrm{m}$, $d_{p 2}=350 \mu \mathrm{m}$, and $d_{p 3}=600 \mu \mathrm{m}$. (The need for adequate air-grain coupling sets an upper grain size limit of $600 \mu \mathrm{m}$.$) .$

\section{Polydisperse Aggregates}

In a polydisperse aggregates, both slipping and sticking motions can be observed. The sticking motion could cause the aggregates to fold and consequently become (considerably) more compact. 
Consider a simple granular aggregate consisting of three spheres with diameters of $d_{p 1}=500 \mu \mathrm{m}, d_{p 2}=350 \mu \mathrm{m}$, and $d_{p 3}=600 \mu \mathrm{m}$. Fig. 3(a) represents a sketch of the aggregate and the grid. The grid is built sufficiently fine in regions of high gradients of stresses and strains. The coefficient of static friction between the spheres is assumed to be $\mu_{s}=0.65$. The coefficient of kinetic friction is assumed to depend on $V_{\text {rel }}$, which is the relative velocity of the surfaces in contact [13]. It decreases with an increase in velocity. The coefficient of kinetic friction may be represented by $\mu_{\varepsilon}=\mu_{d}+\left(\mu_{p}-\mu_{d}\right) e^{-c V_{r a l}}$ where $\mu_{d}$ represents the coefficient of dynamic friction and $C$ is the exponential decay coefficient which represents the unknown quantity in advance; it may be extracted from the data. The coefficient of dynamic friction can be estimated as $\mu_{d}=\tan \left(\theta_{d}\right)$, where $\theta_{d d}$ is the dynamic angle of repose. In this case, the magnitude of $\mu_{d}$ is assumed to be 0.55 .

In this simulation, "ball 1 " with a diameter of $d_{p 1}=500 \mu \mathrm{m}$ and velocity of $V_{x 0}^{(1)}$ collides with the stationary "ball 2" with a diameter of $d_{p 2}=350 \mu \mathrm{m}$ so that rotational motions are induced due to frictional forces between them.

Using Finite Element Methods (FEM), as detailed in [18], time evolutions of the dimensionless velocities of the balls were predicted and depicted in Fig. 3(b). As can be seen from the last configuration in Fig. 3(b), "ball 2" and "ball 3" stuck together at the end of the interaction. Figures $3(\mathrm{c}-\mathrm{d})$ show the time evolution of the translational and angular velocities of the balls in the aggregate. As shown in Fig. 3(d), "ball 2" displaces in the $x$-direction and collides with the stationary "ball 3" with a diameter of $d_{p 3}=600 \mu \mathrm{m}$. It is noteworthy that after the collision the angular velocity of "ball 3" is in the opposite direction of that of "ball 2".

Fig. 4(a-b) represents variations of the dimensionless velocities of the center of mass of balls in the $x$ - and $y$-directions, respectively, as a function of time. Here, and in Fig. 4(c), squares, circles and diamonds represent the dimensionless velocities of "ball 1", "ball 2", and "ball 3", respectively. The dimensionless velocities in the $x$ - and $y$-directions are defined as $V_{x}^{*}=V_{x} / V_{x 0}^{(1)}$, and $V_{y}^{*}=V_{y} / V_{x 0}^{(1)}$, respectively. Fig. $4(\mathrm{~d})$ shows variations of $\omega_{p z}^{*}$, defined as $\omega_{p z}^{*}=\omega_{p z} / V_{x 0}^{(1)}$, where $\omega_{p z}$ represents the particle angular velocity as a function of time.

At this stage, the complex contact dynamics of particles in the simple polydisperse aggregate detailed above is calculated using a simplified model as detailed in [18]. The aim is to assess the reliability of the simplified model in predicting complexities involved in dense granular flows. Fig. 4(b), (d) and (f) depict the predictions of the simplified model [18] for the dynamics of the aforementioned simple aggregate for comparison. As can be seen from Figs. 4 (a-f), the agreement between the FEM results and those predicted by the simplified model [18] is satisfactory, implying that the simplified model is capable of reproducing the observed behaviour by utilizing FEM. 


\section{A Model for the Fluid Phase}

The deviation of pressure below a granular bed of thickness $h$ from the ambient pressure may be given as [19]:

$$
\Delta \mathrm{p}=-h \gamma \rho_{a}(1-\phi) v_{a}
$$

where $\gamma$ describes the grain-air coupling which is introduced through the use of Darcy's law, $\rho_{a}$ is the air density, $\phi$ is the bed porosity, and $v_{a}$ is the air velocity. Darcy's flow is observed for $0.3<\mathrm{Re} \leq 0.7$ [20], where the Darcian Reynolds number is defined as $\operatorname{Re}=\rho_{a} v_{a} d_{p} / \mu_{\mathrm{a}}$. Here, $\mu_{\mathrm{a}}$ is the air dynamic viscosity. The physical properties of the air are given in Table. 1. The flow Reynolds number in the experiments is described in the preceding section is larger than 0.7 . Hence, the flow regime is likely to be turbulent [20]. Note that the granular bed Reynolds number, which is used as the demarcation criterion, is defined as $\operatorname{Re}_{\text {slip }}=\rho_{a}\left|\mathbf{v}_{\mathrm{a}}-\mathbf{V}_{\mathrm{p}}^{\mathrm{i}}\right| d_{p} / \mu_{\mathrm{a}}$, where $V_{p}^{i}$ is the velocity of the $i^{\text {th }}$ particle.

In this attempt the aim is to combine the Lagrangian (particle-based) model, which is briefly described in the preceding section, and the Large eddy simulation (LES) [11], in order to achieve more accurate simulations of the tilting and especially of the swirl of granular flows.

The filtered continuity momentum for an isothermal 3D flow of air can be given as:

$$
\begin{gathered}
\frac{\partial \bar{\rho}}{\partial t}+\nabla \cdot\left(\widehat{\rho} \mathbf{v}_{a}\right)=0 \\
\frac{\partial \bar{\rho} \widetilde{v_{a}}}{\partial t}+\nabla \cdot\left(\widetilde{\rho v_{a} \widetilde{v}_{a}}\right)=-\frac{1}{\rho_{a}} \nabla(\bar{\rho} \bar{p})+\frac{1}{\rho_{a}} \nabla \cdot(\bar{\rho} \tilde{\sigma})+\nabla \cdot \mathbf{\tau}+\overline{\mathbf{f}}_{p f}+\bar{\rho} \mathbf{g}
\end{gathered}
$$

where $\bar{\rho}$ is the filtered density defined as $\bar{\rho}=\rho_{a} \bar{\phi}, \widetilde{\mathbf{v}_{a}}$ is the density-weighted Favre filter velocity defined as $\widetilde{\mathbf{v}}_{a}=\overline{\rho \mathbf{v}_{\alpha}} / \bar{p}, \bar{p}$ is the filtered pressure $\overline{\mathbf{f}}_{p f}$ at the supergrid scale. The forces exerted by the fluid on the particles per unit volume $\overline{\mathbf{f}}_{f p}$ encompass the drag, lifts, added mass, stress, and Basset history forces [21],g is the acceleration of gravity.

The viscous stress tensor in (5) is defined as:

$$
\sigma=-\frac{2}{3} \mu_{a} \nabla \cdot \mathbf{v}_{a} \mathbf{I}+\mu_{a}\left(\nabla \mathbf{v}_{a}+\left(\nabla \mathbf{v}_{a}\right)^{T}\right)
$$

where $\mathbf{I}$ is the identity tensor of second order. In Eq. (5), $\tau$ is the subgrid scale (SGS) stress tensor given as

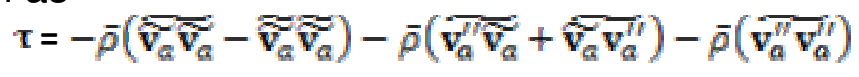

where $\mathbf{v}_{a}^{\prime \prime}$ represents the subgrid-scale part of $\mathbf{v}_{a}$ based on Favre filtering. The SGS stress tensor $\tau$ is required to close the equations for the large scale fields on a grid 
small enough (but much larger than the Kolmogorov scale) to provide reasonable resolutions. In contrast to the filtered single-phase equations, a conceptual restriction arising in the present approach that the filter width $\Delta$ should strictly be larger than the length scale characteristic of the bubble phase. Thus, an appropriate choice of $\Delta$ should provide a sufficiently large-scale resolution while not violating the aforementioned restriction.

It is known that the presence of the particles make a contribution to the processes of energy removal from the resolved scales of the fluid phase. This two-way coupling effect may be modelled by superposing particle-induced SGS energy dissipation to that induced by shear. A tentative first attempt at closure, the momentum equation (4) may be closed with an SGS model for, $\tau$, given as:

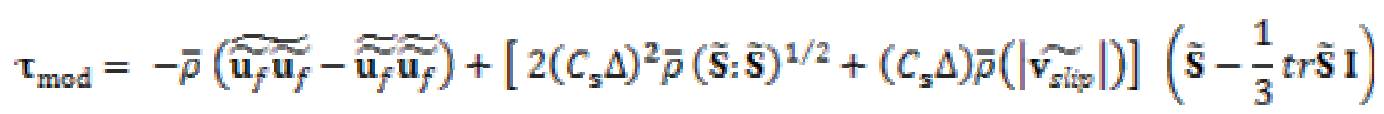

where $C_{s}$ is the Smagorinsky constant, given as $C_{s}=2 \pi / 3 \sqrt{3}\left(-R\left(1+C_{b 0} R^{2}\right) / K^{3}\right)^{\frac{\pi}{2}}$, $\mathrm{R}$ is related to the ratio of the mesh spacing and the Kolmogorov length scale, so that $\mathrm{R}=-1+(\mathrm{n} / \Delta)^{4 / 3}, \mathrm{~K}$ is the Kolmogorov constant, $\mathrm{C}_{\mathrm{bo}}$ is the backscatter parameter with a value set to $\mathrm{C}_{\mathrm{bo}}=0.2, \overline{\mathrm{s}}$ is the resolved rate of strain tensor, and $\mathrm{v}_{\text {slip }}$ represents the slip velocity.

\section{Simulation of Shaken Sand by an Orbital Shaker}

In this section, simulations of a bed of air-immersed solid particles inside a polycarbonate plastic container are being performed by using the models described in the preceding sections.

A solid mixture consists of spherical particles with diameters of $d_{p m}=500 \mu \mathrm{m}$, $d_{p s}=350 \mu \mathrm{m}$, and $d_{p l}=600 \mu \mathrm{m}$. The mixture was initially poured into the container to a height of $h_{\mathrm{p} 0}$, as shown in Fig. 5 (a). The volume fraction of the smallest particle size in the mixture was $\phi_{s z}=0.20$. The total solid volume fraction in the container was $\phi_{s}=0.64$ and the solid volume fraction of the largest particles was $\phi_{s l}=0.18$.

The free surface of the solid particles before they were exposed to horizontal oscillations was nearly flat. The container is exposed to orbital (horizontal) oscillations of the form of equation (1) where $\omega_{x} / 2 \pi=42 \mathrm{~Hz}$ and $A_{x}=10^{-3} \mathrm{~m}$ and $\omega_{y} / 2 \pi=25 \mathrm{~Hz}$, and $A_{y}=10^{-3} \mathrm{~m}$.

Figure 5 (b) shows the tilted beads of the solid particles until the tilt angle reached a dynamic angle of repose, $\theta_{d}$, at time $t=1 \mathrm{sec}$. Figure 3 (c) illustrates the configuration of solid particles at time $t=2 \mathrm{sec}$. Figure 3 (d) depicts the computed instantaneous average velocity field of the particle on the $y^{\prime} z^{\prime}$-plane, the position is shown in the inset. The inset also shows the computed free surface of the airparticle. The free surface with a tilt angle of $\theta_{d}$ is also highlighted in Fig. 3 (d). Here, the computed dynamic angle of repose is found to be $\theta_{d}=29^{\mathrm{a}}$. 
By comparing the results shown in Fig. 1 (a) and Fig. 3 (c), it may be concluded that the model is sufficiently flexible to capture a number of salient features of experiments. Note that the granular bed Reynolds number is found to be larger than 50 . This finding justifies the use of LES for simulations of the tilting granular flows.

\section{Simulation of Swirl Granular Flows}

Figure $6(a-b)$ illustrate initial configuration of sand particles which are exposed to horizontal vibrations. Similar to the case described in the preceding section, a solid mixture of spherical particles with diameters of $d_{p m}=500 \mu m, d_{p s}=350 \mu \mathrm{m}$, and $d_{p l}=600 \mathrm{\mu m}$ was used in the simulation. The volume fractions of the smallest, medium and largest particle size in the mixture were $\phi_{s s}=0.20, \phi_{s m}=0.26$ and $\phi_{s l}=0.18$, respectively.

Figure 7 (a-c) shows an instantaneous configuration of the particles after $t=2$ sec. Figure 7 (d) depicts the computed instantaneous average velocity field of the particle on the $x y$-plane at the same position. A simple analysis of Fig. 7 (d) reveals that the instantaneous spin of the particles is $\omega_{p}=15.7 \mathrm{rad} / \mathrm{s}$. The spin has been found to be nearly constant so that the system, combined with the mathematical model applied in this study, has a potential to be used as a viscometer for dense granular fluids. Figure 7 (e) shows the computed instantaneous average velocity field of the particle on the $x z$-plane. This indicates a novel swirl-convection regime for a granular. Figure 7 (f) illustrates the computed instantaneous average velocity field of air on the $x y$ plane like that in Fig. 7 (d). As can be seen from Fig. 7 (f), the air is being displaced in the negative $x$-direction due to the instantaneous acceleration of the container. However, the displacement of solid particles appears to be small, as shown in Fig. 7 (d).

A large granular bed Reynolds number computed for the swirl-convection regime indicates that the use of LES is necessary for accurate simulations of complex granular flows.

\section{CONCLUSIONS}

A horizontally vibrated air-grain system exhibits a novel swirl granular flow at the axial and lateral frequencies $\omega_{x} / 2 \pi=42 \mathrm{~Hz}$ and $\omega_{y} / 2 \pi=25 \mathrm{~Hz}$, respectively. The granular bed Reynolds number was found to be in the range of $25<\mathrm{Re}_{\text {slip }} \leq 18$ which indicates that the flow regime is turbulent. Results from the experiments indicate an advantage to use Large Eddy Simulation for modelling of fluid flow. The observed swirl-convection regime in experiments was successfully simulated by using a Lagrangian (particle-based) method combined with LES. Hence, the model (Eu/La) presented in this work provides an appropriate mathematical framework for investigating dense granular flows. In addition, an optical processing algorithm was developed that employs numerous photographs taken at multiple known fixed locations at approximately the same time to create a 3 dimensional model of the system. This image processing algorithm combined with the $\mathrm{Eu} / \mathrm{La}$ mathematical methods running on GPUs will become interesting in the field of rheology of dense granular fluids. 


\section{REFERENCES}

[1] Hough, S., Earthshaking Science: What We Know (and Don't Know) about Earthquakes, Princeton University Press, New Jersey, (2002).

[2] Pudasaini, S. P. and Hutter, K., Avalanche dynamics, Springer, Berlin, (2007).

[3] Gutman, I., Industrial Uses of Mechanical Vibrations, Beekman Pub, London, (1968).

[4] Orpe, A. V., "Solid-Fluid Transition in a Granular Shear Flow", The American Physical Society, (2004).

[5] Metcalfe, G., Tennakoon, S. G. K., Kondic, L., Schaeffer, D. G. and Behringer, R. P., "Granular friction, Coulomb failure, and the fluid-solid transition for horizontally shaken granular materials", PHYSICAL REVIEW E; VOLUME 65 (2002)

[6] Rubin, D., Goldenson, N. and Voth, G. A., "Failure and strengthening of granular slopes under horizontal vibration", PHYSICAL REVIEW E, |28 November|; VOLUME 74, (2006).

[7] Aumaitre, S., Puls, C., McElwaine, J. N. and Gollub, J. P., "Comparing flow thresholds and dynamics for oscillating and inclined granular layers", PHYSICAL REVIEW E, |27 June|; VOLUME 75, (2007).

[8] Bejan, A. and Kraus, A.D., Heat transfer handbook, Wiley, New Jersey, (2003).

[9] Anderson, T. B. and Jackson, R., "Fluid mechanical description of fluidized beds", Ind. Eng. Chem. Fundam. 7 (1)I 12-21 (1968).

[10] Lemieux, P.-A. and Durian, D. J., From avalanches to fluid flow: A continuous picture of grain dynamics down a heap, Phys. Rev. Lett. 85 (20) 4273-4276 (2000).

[11] Sagaut, P., Large Eddy Simulation for Incompressible Flows, Springer, Berlin, (1998).

[12] Zamankhan, P., "Simulation of self-segregation of a low density spherical particle in a bubbling bed", Chemical Engineering Journal, DOI: 10.1016/j.cej. 2011.11.092. (2011).

[13] Rabinowicz, E., Friction and Wear of Materials, Wiley, New York, (1965).

[14] http://www.nvidia.com/object/GPU_Computing.html.

[15] Bai, G. and Bee, J. S., "Computational fluid dynamics (CFD) insights into agitation stress methods in biopharmaceutical development", International Journal of Pharmaceutics, DOI: 10.1016/j.ijpharm.2011.11.044, (2011).

[16] Colomer, J., Petersb, F. and Marrase, C., "Experimental analysis of coagulation of particles under low-shear flow", Water Res., 39 , pp. 2994-3000, (2005).

[17] ASTM International - Standards Worldwide. ASTM C136-06. http://www.astm.org/cgibin/SoftCart.exe/DATABASE.CART/REDLINE PAGES/C1 36.htm, (2006).

[18] Zamankhan, P. and Bordbar, M-H, "Complex Flow Dynamics in Dense Granular Flows. Part I: Experimentation", J. Appl. Mech. (T-ASME), 73, 648-657, (2006)

[19] King, P.J., Lopez-Alcaraz, P., Pacheco-Martinez, H.A., Clement, C. P., Smith, A. J., and Swift, M.R., "Instabilities in vertically vibrated fluid-grain systems", Eur. Phys. J. E 22, 219 (2007).

[20] Jafari, A., Zamankhan, P., Mousavi, S. M. and Pietarnen, K., "Modelling and CFD simulation of flow behaviour and dispersivity through randomly packed bed reactors," Chemical Engineering Journal, 144, 476 (2008).

[21] Maxey, M. R., and Riley, J. J., "Equation of motion for a small rigid sphere in a nonuniform flow", Physics of Fluids, 26, 883 (1983).

[22] Serini, V. "Polycarbonates" in Ullmann's Encyclopaedia of Industrial Chemistry, Wiley-VCH, Weinheim, (2000). 
[23] Lekhnitskii, SG., "Theory of elasticity of an anisotropic elastic body", Holden-Day Inc. , (1963).

[24] Bandyopadhyay, A. and Basak, C., "Studies on photocatalytic degradation of polystyrene". Materials Science and Technology 23 (3): 307-317, (2007).

(a)

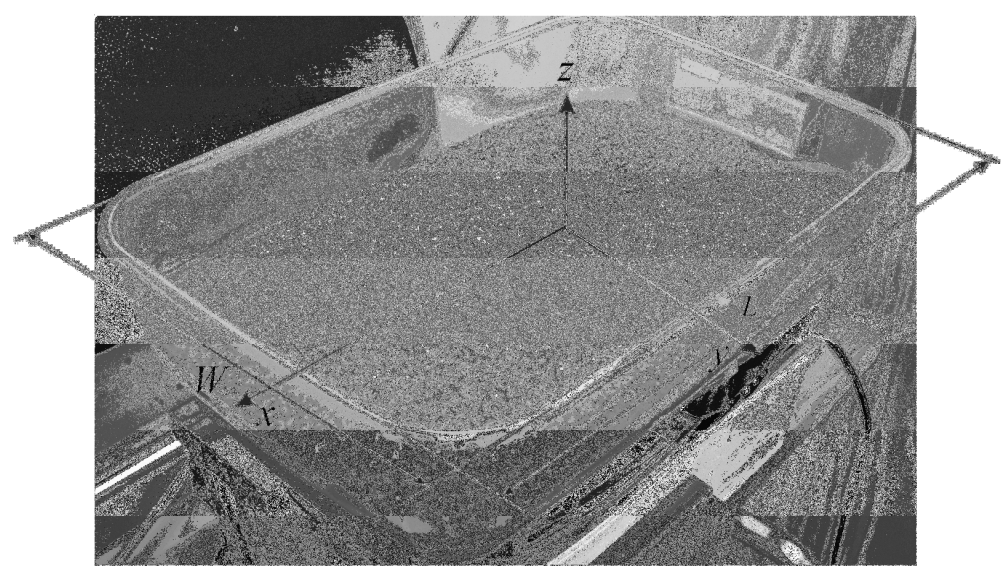

(b)

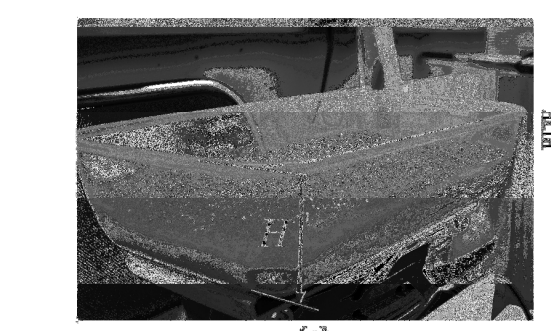

(e)

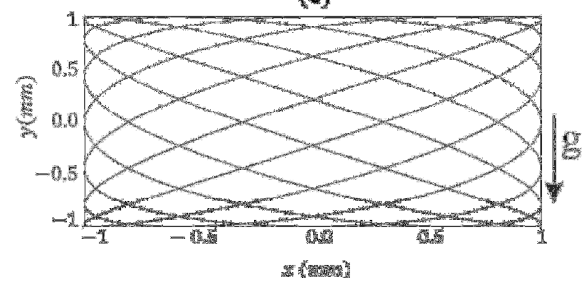

(c)

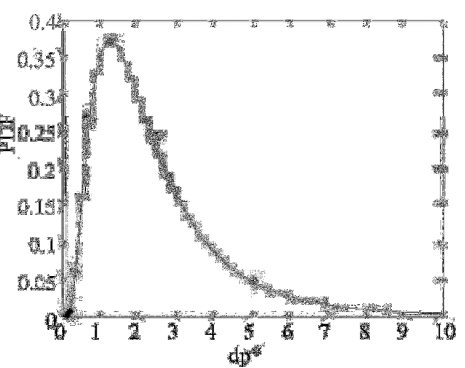

(d)

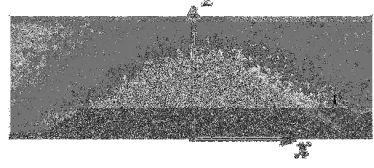

Fig.1. (a) and (b) Images of the container as well as some nomenclatures. Here, $W=18 \mathrm{~cm}$, $\mathrm{L}=28 \mathrm{~cm}$ and $\mathrm{H}=7 \mathrm{~cm}$. (c) The particle size distribution in sand used in the experiments. The circles represent the result of a sieve analysis. The solid line is a log-normal fit with the location parameter and the scale parameter of $\mu=0.69$ and $\sigma=$ 0.68 , respectively. (d) An image of a spill of a ternary mixture of glass beads used in the experiments onto a flat surface. The slope of the pile is $\theta_{s}=34^{\circ}$. (e) The typical ellipse-type orbits of the shaker. 
(a)

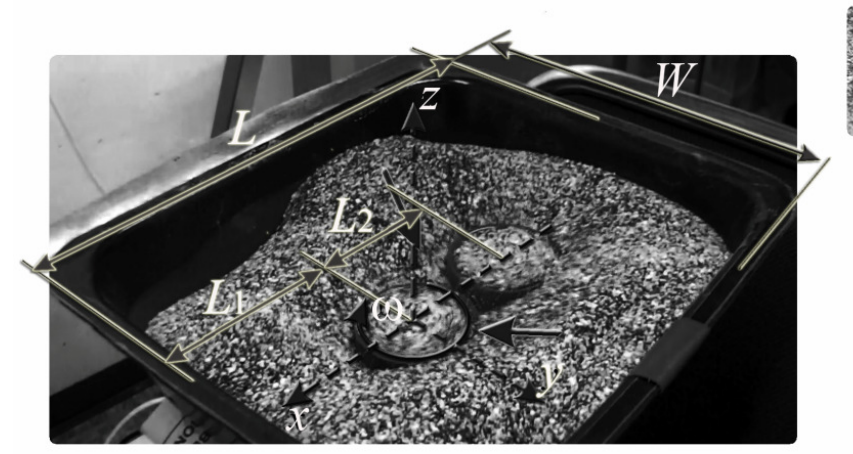

(b)

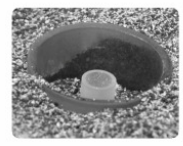

(c)

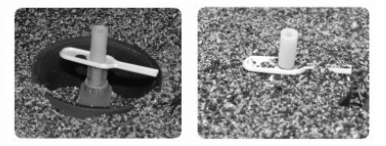

(e)

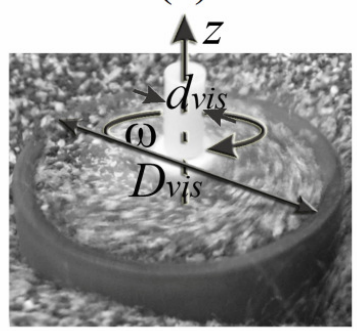

Fig.2. (a) An image of the black foam tray with two hallow cylinders made out of glass inside of the tray. Here, $W=22 \mathrm{~cm}, L=24 \mathrm{~cm}, L_{1}=9 \mathrm{~cm}$ and $L_{2}=6$. (b-d) The propeller and shaft system used to measure the spin of granular material. (e) A snapshot of the circulatory granular flow. Here, $D_{\text {vis }}=2.5 \mathrm{~cm}$.

(a)

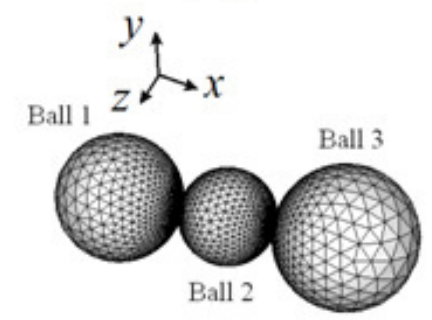

(b)

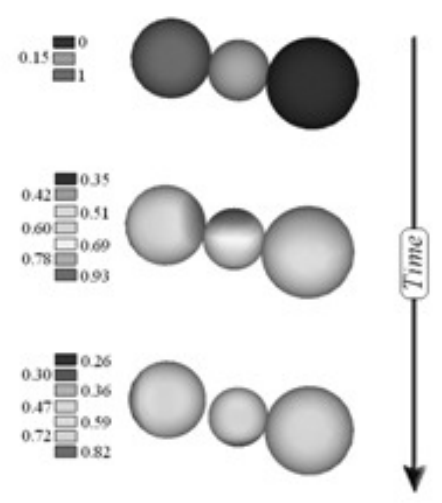

(c)
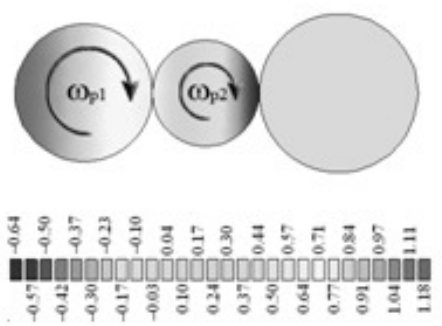

(d)
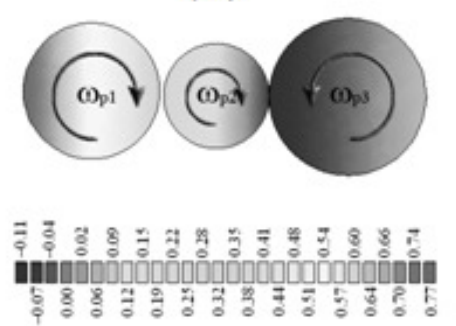

Fig.3. (a) A polydisperse aggregate and its computational grid. The diameter of ball1, ball2 and ball 3 are $500 \mu \mathrm{m}, 350 \mu \mathrm{m}$, and $600 \mu \mathrm{m}$, respectively. (b) Time evolutions of the dimensionless velocities of the particles in the aggregate. Note that at the end of contact processes, ball2 and ball3 were stuck together. (c) and (d) Time evolutions of the translational and angular velocities of the particles in the aggregate. The two configurations are separated by $t=20 \mu \mathrm{sec}$. 
(a)

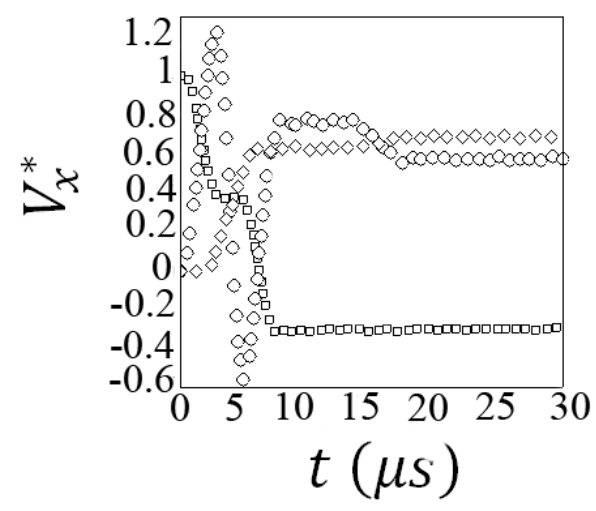

(c)

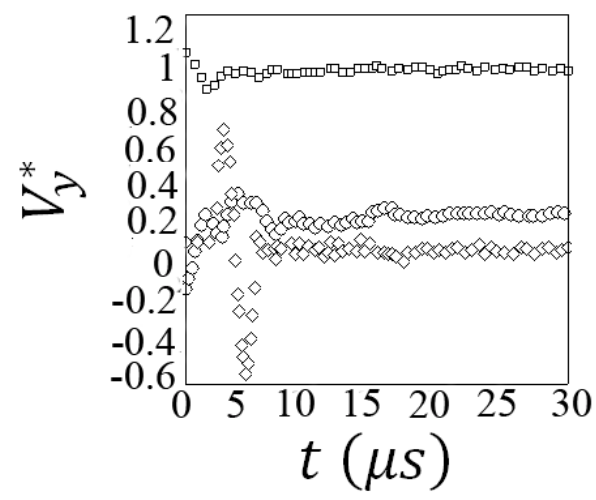

(e)

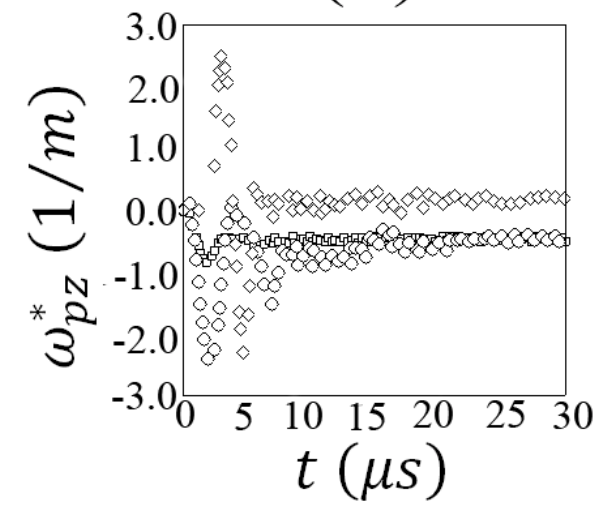

(b)

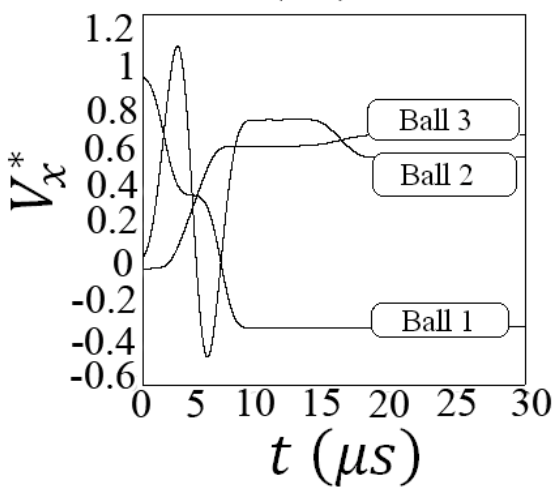

(d)

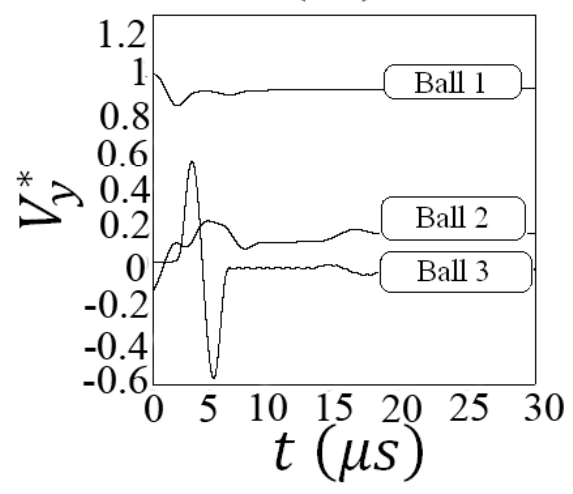

(f)

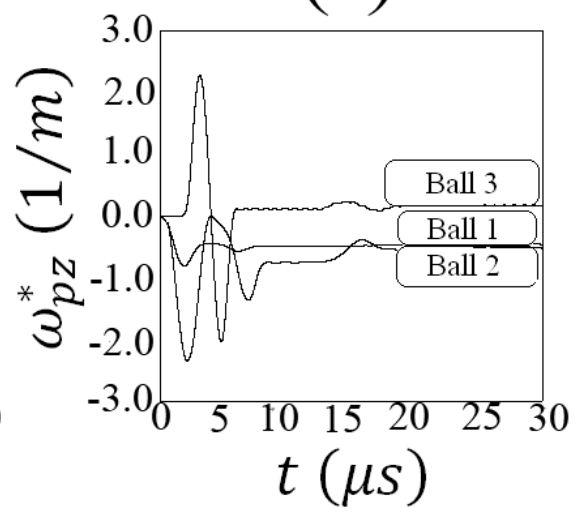

Fig.4. (a) Variations of the computed dimensionless velocity of particles in the $x$ - direction using FEM as a function of time. Here and in (b) and (e), squares, circles and diamonds represent the dimensionless velocities of "ball 1", "ball 2" and "ball 3" respectively. (b) Variations of the computed dimensionless velocity of particles in the $y$-direction using FEM as a function of time. (c) Variations of the computed angular velocity of particles in the $z$ - direction using FEM as a function of time. (d) Variations of the computed dimensionless velocity of the particles in the $x$ direction as a function of time. Here and in (e) and ( $f$ ), the results are obtained by using the simplified model. (e) Variations of the computed dimensionless velocity of particles in the $y$ direction as a function of time. (f) Variations of the computed angular velocity of particles in the $z$ - direction as a function of time. 
(a)

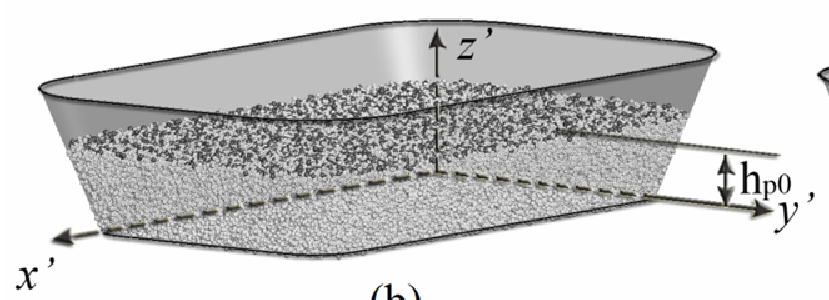

(b)
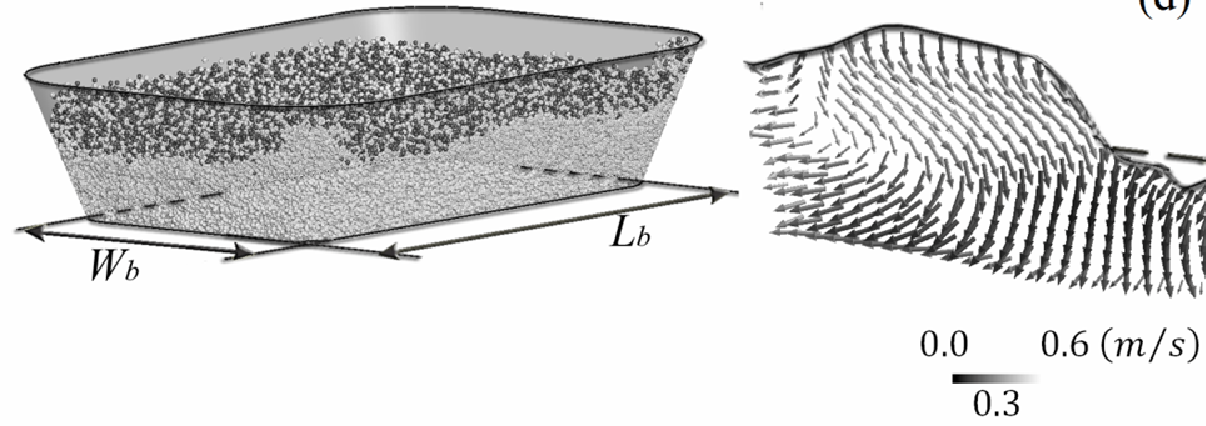

Fig.5. (a) The initial configuration of the solid particles used for numerical simulation. Here, $h_{\mathrm{p} 0}=3.5$ $\mathrm{cm}$. (b) An instantaneous configuration of the solid particles at $t=1 \mathrm{sec}$. Here, $W_{\mathrm{b}}=14 \mathrm{~cm}$ and $L_{b}=24$. (c) An instantaneous configuration of the solid particles at $t=2 \mathrm{sec}$. (d) the computed instantaneous particle average velocity field on a cutting $y^{\prime} z$ '-plane. Inset: The free surface and he position of the cutting plane. Here, $L_{s}=12$.

(a)

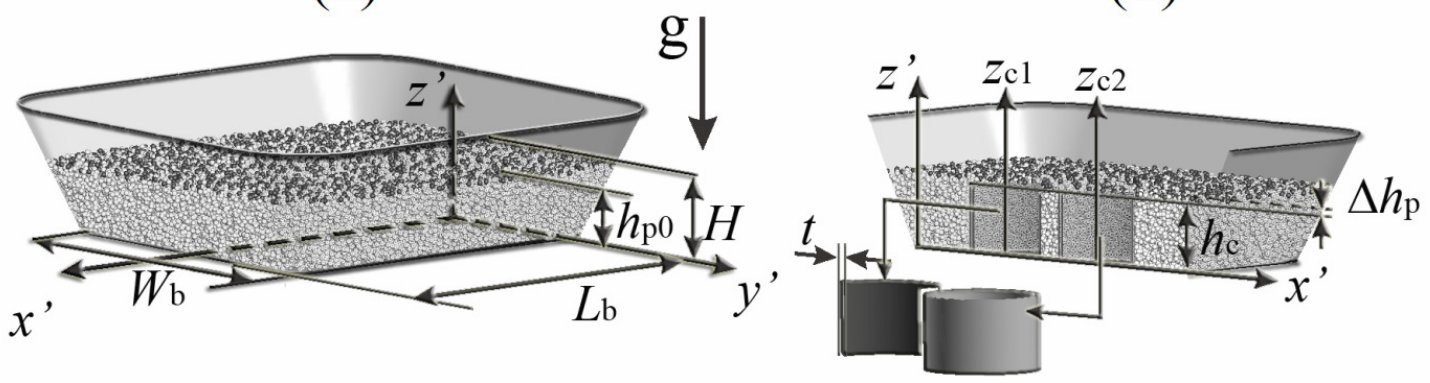

Fig.6. (a) and (b) The initial configuration of the solid particles used for numerical simulation. Here, $h_{\mathrm{p} 0}$ $=3.5 \mathrm{~cm}, H=6 \mathrm{~cm}, W_{\mathrm{b}}=14 \mathrm{~cm}, L_{\mathrm{b}}=16 \mathrm{~cm}, h_{\mathrm{c}}=3.2 \mathrm{~cm}, t=0.2 \mathrm{~cm}$ and $\square h_{\mathrm{p}}=0.3 \mathrm{~cm}$. 
(a)
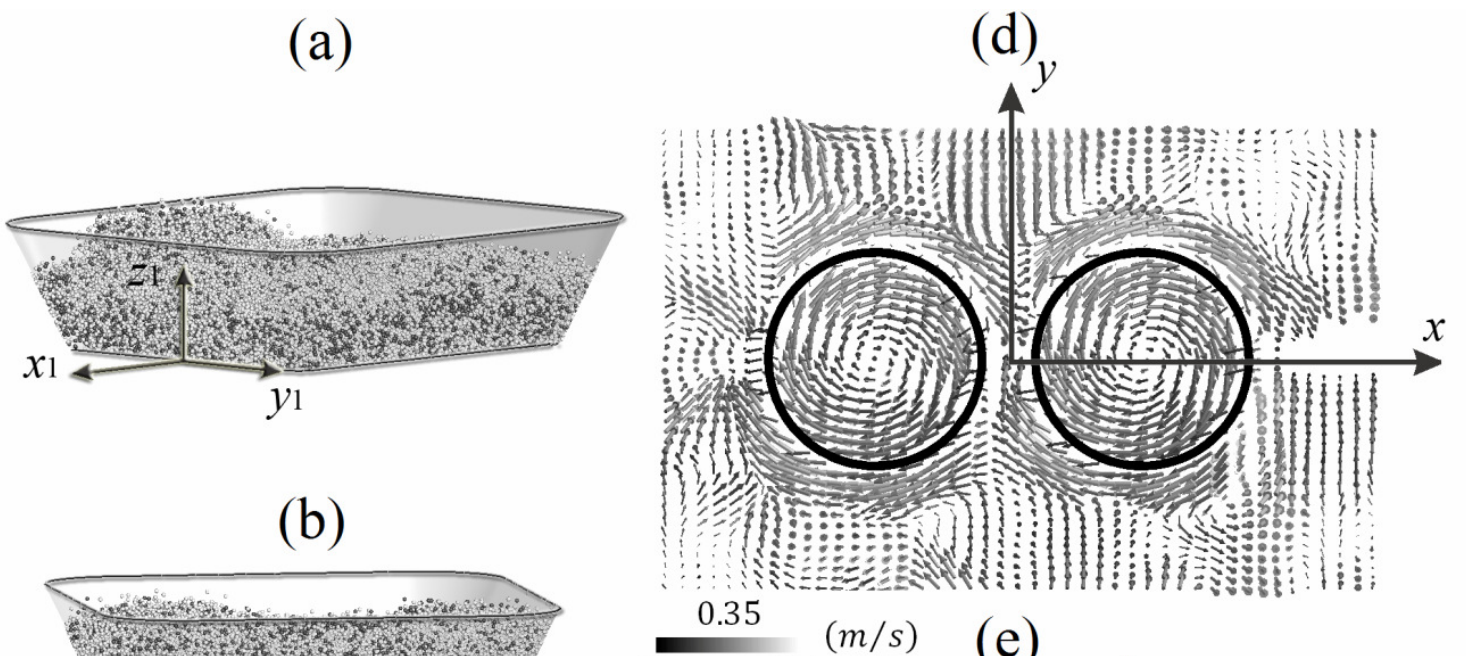

(b)

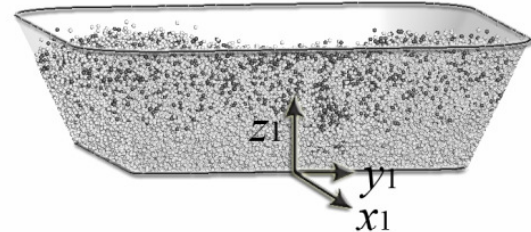

(c)
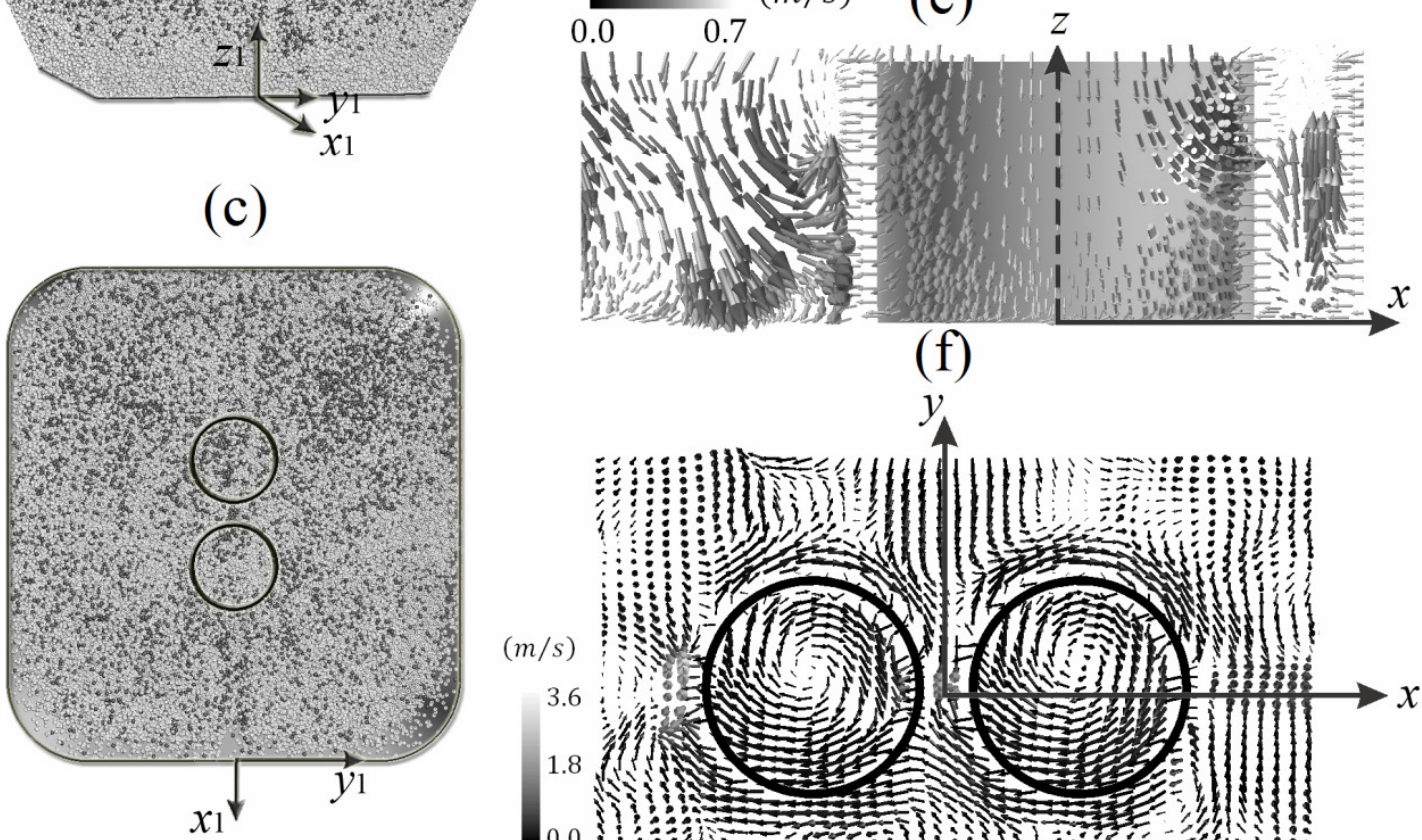

(f)

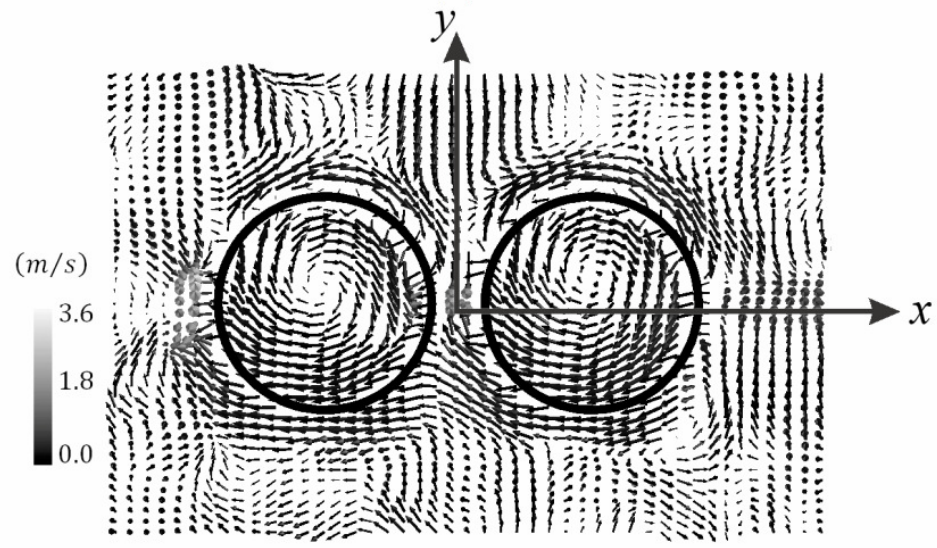

Fig.7. (a-c) An instantaneous configuration of the solid particles at $t=2 \mathrm{sec}$. (d) The computed instantaneous particle average velocity field on the $x y$-plane located at $z_{1}=3.22 \mathrm{~cm}$. (e) The computed instantaneous particle average velocity field on the $x z$-plane located at $y_{1}=0$. (f) The computed instantaneous air average velocity field on the same $x y$-plane as in (d). 
Table 1. Material Properties [22-23-24].

\begin{tabular}{|c|c|c|c|}
\hline Material & Property & Symbol & Value \\
\hline \multirow{5}{*}{$\begin{array}{l}0 \\
\frac{0}{\pi} \\
\frac{1}{0} \\
\frac{0}{2} \\
\frac{0}{\pi} \\
\frac{0}{2} \\
0 \\
0\end{array}$} & Density & $\rho_{p}$ & $1.20-1.22 \mathrm{~g} / \mathrm{cm} 3$ \\
\hline & Young's modulus & $\mathrm{E}$ & 2.0-2.4 GPa \\
\hline & Tensile strength & $\sigma_{i}$ & $55-75 \mathrm{MPa}$ \\
\hline & Poisson's ratio & $v$ & 0.37 \\
\hline & Coefficient of friction & $\mu$ & 0.31 \\
\hline \multirow{5}{*}{$\begin{array}{l}\tilde{\mathscr{N}} \\
\frac{\pi}{0}\end{array}$} & Elastic modulus & $\mathrm{E}$ & $6.3 \times 10^{10} \mathrm{~Pa}$ \\
\hline & Density & $\rho_{p}$ & $2390 \mathrm{~kg} / \mathrm{m}^{3}$ \\
\hline & Poisson's ratio & $v$ & 0.244 \\
\hline & Instantaneous shear modulus & $G_{0}$ & $2.53 \times 10^{10} \mathrm{~kg} / \mathrm{ms}$ \\
\hline & Long time shear modulus & $G_{\infty}$ & $6.1 \times 10^{9} \mathrm{~kg} / \mathrm{ms}$ \\
\hline \multirow{3}{*}{ 妾 } & Density & $\rho_{g}$ & $1.225 \mathrm{~kg} / \mathrm{m}^{3}$ \\
\hline & Kinematics Viscosity & $v_{g}$ & $1.7894 \times 10^{-5} \mathrm{~kg} / \mathrm{ms}$ \\
\hline & Temperature & $T_{g}$ & $293 \mathrm{~K}$ \\
\hline \multirow{4}{*}{$\begin{array}{l}\bar{D} \\
\text { त } \\
\sim\end{array}$} & Poisson's ratio & $v$ & 0.17 \\
\hline & Density & $\rho_{g}$ & $2.203 \mathrm{~g} / \mathrm{cm}^{3}$ \\
\hline & Bulk modulus & K & $37 \mathrm{GPa}$ \\
\hline & Young's modulus & $\mathrm{E}$ & $71.7 \mathrm{GPa}$ \\
\hline \multirow{3}{*}{ 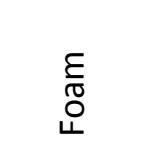 } & Poisson's ratio & $v$ & 0.1 \\
\hline & Density & $\rho_{g}$ & $1.05 \mathrm{~g} / \mathrm{cm}^{3}$ \\
\hline & Young's modulus & $E$ & $3000 \mathrm{MPa}$ \\
\hline
\end{tabular}

